\title{
Airway morphometry in COPD with bronchiectasis: a view on all airway generations
}

\author{
Stephanie Everaerts (1) ${ }^{1,2}$, John E. McDonough ${ }^{1}$, Stijn E. Verleden¹, \\ Iván Josipovic ${ }^{3}$, Matthieu Boone ${ }^{3}$, Adriana Dubbeldam4, Carolien Mathyssen ${ }^{1}$, \\ Jef Serré ${ }^{1}$, Lieven J. Dupont ${ }^{1,2}$, Ghislaine Gayan-Ramirez ${ }^{1}$, \\ Johny Verschakelen ${ }^{4}$, James C. Hogg ${ }^{5}$, Geert M. Verleden ${ }^{1,2}$, \\ Bart M. Vanaudenaerde ${ }^{1}$ and Wim Janssens (1) ${ }^{1,2}$
}

\begin{abstract}
Affiliations: 'Laboratory of Pneumology, Dept of Chronic Diseases, Metabolism and Aging (CHROMETA), KU Leuven, Leuven, Belgium. ${ }^{2}$ Dept of Respiratory Diseases, University Hospitals Leuven, Leuven, Belgium. ${ }^{3}$ Dept of Physics and Astronomy, UGCT, Radiation Physics, Ghent University, Gent, Belgium. ${ }^{4}$ Dept of Radiology, University Hospitals Leuven, Leuven, Belgium. ${ }^{5}$ University of British Columbia James Hogg Research Centre, St Paul's Hospital, Vancouver, BC, Canada.
\end{abstract}

Correspondence: Wim Janssens, University Hospitals Leuven, Dept of Respiratory Diseases, Herestraat 49, B-3000 Leuven, Belgium. E-mail: wim.janssens@uzleuven.be

@ERSpublications

Although terminal bronchioles are equally reduced in COPD lungs with and without bronchiectasis, significantly more large and small airways are found in COPD lungs with bronchiectasis http://bit.ly/2YJ5kKs

Cite this article as: Everaerts S, McDonough JE, Verleden SE, et al. Airway morphometry in COPD with bronchiectasis: a view on all airway generations. Eur Respir J 2019; 54: 1802166 [https://doi.org/10.1183/ 13993003.02166-2018].

ABSTRACT The pathophysiological processes underlying bronchiectasis in chronic obstructive pulmonary disease (COPD) are not understood. In COPD, both small and large airways are progressively lost. It is currently not known to what extent the different airway generations of patients with COPD and bronchiectasis are involved.

COPD explant lungs with bronchiectasis were compared to COPD explant lungs without bronchiectasis and unused donor lungs as controls. In order to investigate all airway generations, a multimodal imaging approach using different resolutions was conducted. Per group, five lungs were frozen $(n=15)$ and underwent computed tomography (CT) imaging for large airway evaluation, with four tissue cores per lung imaged for measurements of the terminal bronchioles. Two additional lungs per group ( $\mathrm{n}=6)$ were air-dried for lobar microCT images that allow airway segmentation and three-dimensional quantification of the complete airway tree.

COPD lungs with bronchiectasis had significantly more airways compared to COPD lungs without bronchiectasis $(\mathrm{p}<0.001)$, with large airway numbers similar to control lungs. This difference was present in both upper and lower lobes. Lack of tapering was present $(p=0.010)$ and larger diameters were demonstrated in lower lobes with bronchiectasis $(p=0.010)$. MicroCT analysis of tissue cores showed similar reductions of tissue percentage, surface density and number of terminal bronchioles in both COPD groups compared to control lungs.

Although terminal bronchioles were equally reduced in COPD lungs with and without bronchiectasis, significantly more large and small airways were found in COPD lungs with bronchiectasis.

This article has supplementary material available from erj.ersjournals.com

Received: 13 Nov 2018 | Accepted after revision: 3 Aug 2019

Copyright OERS 2019 


\section{Introduction}

Chronic obstructive pulmonary disease (COPD) is a prevalent disease, characterised by persistent respiratory symptoms and caused by an abnormal inflammatory response to chronic inhalation of irritants. Chronic inflammation affects small airways and lung parenchyma, resulting in progressive airflow limitation [1] with both environmental and host factors contributing to the pathogenesis and heterogeneity of the disease [2,3]. Lately, the overlap between COPD and bronchiectasis has been receiving more attention as a potential COPD phenotype. Bronchiectasis is defined by permanently dilated and chronically inflamed middle-order bronchi, due to a vicious circle of transmural infection and inflammation. This translates into a clinical syndrome with productive cough, bacterial colonisation and recurrent respiratory infections. The diagnosis of bronchiectasis is primarily based on imaging, with the gold standard being abnormal bronchial dilation on $1 \mathrm{~mm}$ computed tomography (CT) images [4]. Bronchodilation is assessed using Naidich's criteria: a broncho-arterial ratio $>1$, lack of tapering and the presence of bronchi within $1 \mathrm{~cm}$ of costal pleura or abutting the mediastinal pleura [5].

The reported prevalence of bronchiectasis in COPD cohorts varies between $4 \%$ and $70 \%$, due to differences in study population and radiological criteria [6]. Conversely, COPD is present in 3-35\% of patients in bronchiectasis cohorts [7-9]. Importantly, patients with COPD and bronchiectasis have a more severe disease course with higher mortality and an obvious need for adjusted therapeutic strategies [9-13]. It is still unclear whether COPD facilitates bronchiectasis or if these two conditions coexist in an individual patient.

Small airways, with a luminal diameter $<2 \mathrm{~mm}$, are the major site of COPD pathogenesis and the main reason for airflow obstruction. It has been demonstrated that the numbers of small airways in COPD are reduced and that terminal bronchioles are narrowed in comparison with controls, even before the onset of emphysema [14, 15]. Moreover, remodelling of small airways has been shown from early disease onwards [16]. More recently, a reduced total airway count on CT has been demonstrated in patients with mild COPD compared to never-smokers and smokers without COPD, suggesting that the large airways may also play a role in pathogenesis [17]. In bronchiectasis, it is assumed that obliteration occurs in the small airways, causing airflow obstruction $[18,19]$. So far, no advanced imaging studies in bronchiectasis have focused on airway generations beyond the resolution of conventional clinical CT. MicroCT imaging, with its increased resolution, is therefore a promising tool enabling evaluation up to the level of the most distal conducting airways or terminal bronchioles [15].

We provide, for the first time, a detailed morphometric analysis of the entire bronchial tree in end-stage COPD lungs with and without bronchiectasis and controls. In 14 COPD explant lungs and seven unused donor lungs, we used a three-fold imaging approach with high-resolution CT $(\sim 600 \mu \mathrm{m}$ resolution), whole-lobe microCT $(\sim 140 \mu \mathrm{m}$ resolution) and microCT scanning of tissue cores $(\sim 10 \mu \mathrm{m}$ resolution $)$ to investigate large and small airways up to the level of the terminal bronchioles.

\section{Materials and methods}

Study material

Explanted lungs of patients with end-stage COPD undergoing lung transplantation and of unused donors were prospectively collected in our centre after approval of the local medical ethics board of University Hospitals Leuven, Belgium (ML6385, s52174). Patients were eligible for lung transplantation based on criteria proposed by the International Society for Heart and Lung Transplantation [20], and gave written informed consent to use their lungs for research purposes. According to Belgian law, declined organs from prospective donors can be used for research. The cause of donor death and reason for lung decline are summarised in the supplementary material (table E1). The donor lungs were inspected carefully during processing to make sure that there was no macroscopic disorder. The presence and morphology of bronchiectasis in COPD lungs was evaluated per lobe on clinical, pretransplant inspiratory CT scans of the lungs using the modified Reiff score (lingula considered separately, tubular $=1$, varicose $=2$ or cystic $=3$ [21]) and confirmed by experienced chest radiologists (AD, JV). None of the patients had a history of bronchiectasis before the diagnosis of COPD and a thorough pretransplant work-up could not reveal a specific cause for bronchiectasis. Based on availability and conservation, five COPD lungs with bronchiectasis, five COPD lungs without bronchiectasis and five unused donor lungs were analysed retrospectively (figure 1a) and two lungs from each group were collected prospectively (figure 1b). COPD lungs without bronchiectasis are referred to as COPD lungs and unused donor lungs as controls hereafter.

Frozen whole-lung CT and tissue core microCT

15 explant lungs, obtained from 15 different individuals, were inflated and frozen as previously described $[15,22]$. A CT scan was obtained from the frozen lungs, resulting in images with a resolution of $\sim 600 \mu \mathrm{m}$ (figure 1a). Emphysema was quantified as the percentage of lung area <-950 HU using customised 


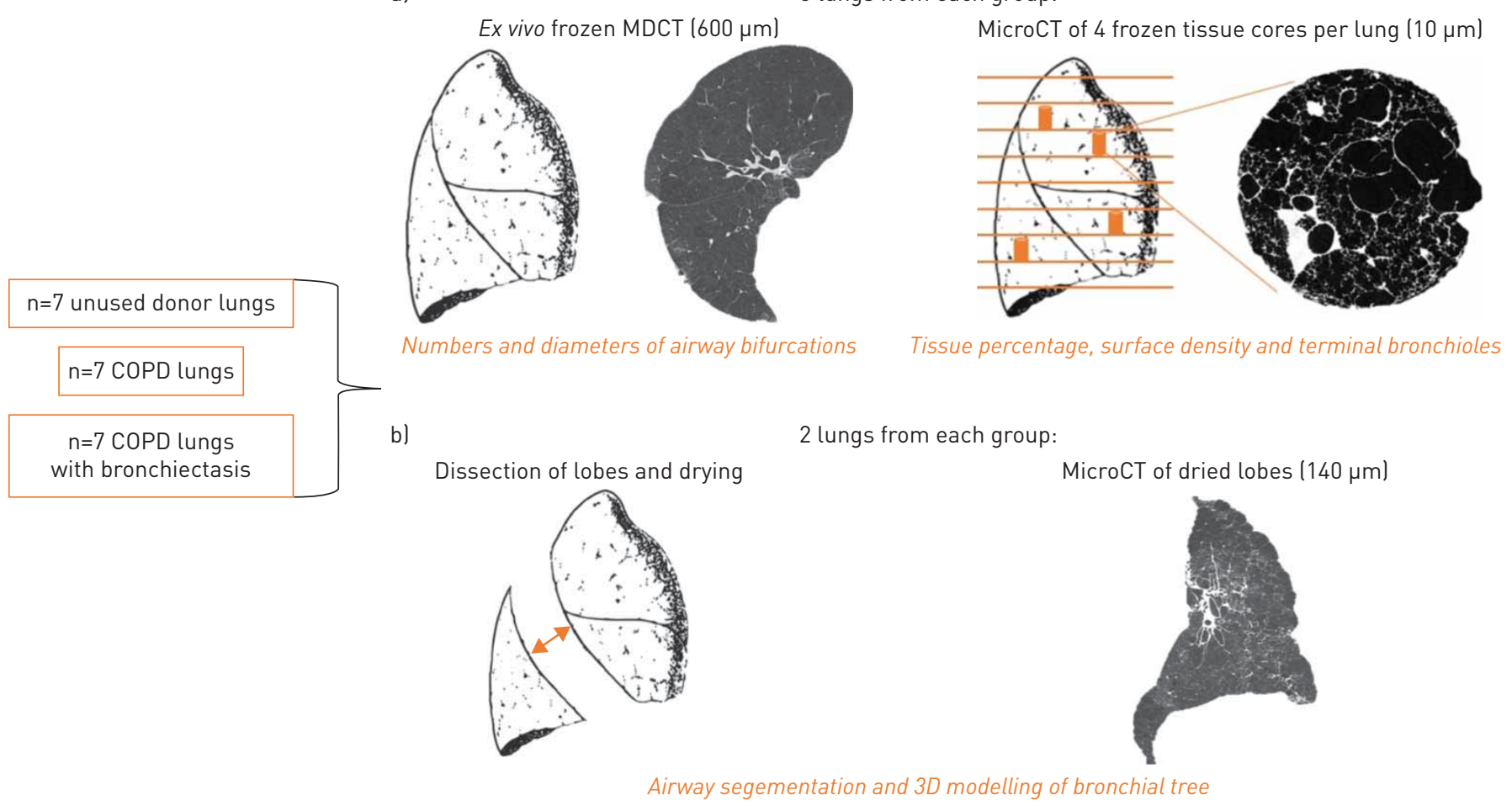

FIGURE 1 Overview of used materials and methods. 21 explant lungs were collected, comprising seven unused donor lungs, seven chronic obstructive pulmonary disease (COPD) lungs and seven COPD lungs with bronchiectasis. a) Of each group, five lungs of five different individuals were prepared for freezing and computed tomography (CT) scanning, and further processed to obtain four tissue cores per lung for microCT. b) Lobes from two lungs per group, obtained from two different individuals, were separated and air-dried to obtain microCT scans of the entire lobes.

in-house software. An open-source image viewer, Horos software version 3.0 (Horosproject; www. horosproject.org) was used for measurements and manual counting of airway bifurcations per generation by scrolling through the ex vivo whole-lung CT images. The airway branches were counted starting from the main stem bronchus (generation 1), reporting each subsequent bifurcation as a new generation and recording the number of visible bifurcations per generation. Inner diameters were measured manually at the widest level in each segment.

After CT scanning, two apical and two basal cylindrical cores $(2 \mathrm{~cm}$ height $\times 1.4 \mathrm{~cm}$ diameter $)$ were obtained from each lung, as previously described [15, 22]. MicroCT scans (Bruker Skyscan 1172; Bruker, Kontich, Belgium) of the frozen tissue cores were performed (10 $\mu \mathrm{m}$ resolution) [23] (figure 1a). Tissue percentage and surface density of the cores were calculated. Decreasing values of surface density reflect loss of normal tissue through disease (e.g. emphysema in COPD). Terminal bronchioles, the last segments of conducting airways before alveolar buds become visible, were anatomically identified, counted and measured on the microCT images.

\section{Air-dried whole-lobe microCT}

Two lungs from each group, obtained from two different individuals, were processed in order to obtain microCT images of entire lobes (figure 1b). Lung lobes rather than whole lungs were scanned due to limitations on the volume of tissue that can be imaged within a single scan. Lobes were separated, cannulated and air-dried for 7 days while continuously inflated at near total capacity. In case of right-sided lungs, the middle lobe was processed as part of the upper lobe. Lobes were scanned with a custom microCT set-up developed at the Ghent University Centre for X-ray Tomography [24], resulting in images with a resolution of $\sim 140 \mu \mathrm{m}$ (figure 1b). Additional detail on this set-up is provided in the supplementary material. Volume of the air-dried lobes and percentage of area <-950 HU was obtained using StratX $\mathrm{X}^{\oplus}$ Lung Analysis Platform (PulmonX, Neuchâtel, Switzerland) on the in vivo inspiratory CT scans. MicroCT images of the lung lobes were used for semi-automatic airway segmentation by region growing in itk-SNAP software [25], which resulted in three-dimensional models of the bronchial tree. These models were used for automatic tracing and reconstruction in NeuronStudio software which applies deconvolution, skeletonisation and the Rayburst algorithm to result in calculation of airway numbers per generation and average inner diameter per segment [26]. 
Statistics

GraphPad Prism 7.0 (San Diego, CA, USA) was used to calculate differences between groups with MannWhitney or Kruskal-Wallis and Dunn's post hoc test. The numbers of airways per generation were compared using two-way ANOVA with Tukey's post hoc test. Analysis of diameters with two-way ANOVA was limited to the generations that were present in all lungs, and due to the processing of the lungs, diameters of generations 1 and 2 (1-3 in case of lobes), were not considered reliable. MicroCT results of tissue cores were analysed with mixed models using SAS 9.4 (SAS Institute, Cary, NC, USA) with subject as random effect. p-values $<0.05$ were considered significant.

\section{Results}

Numbers and diameters of airways on CT images

Characteristics of subjects of which the lungs were analysed are presented in table 1. All patients suffered from end-stage COPD. The subjects were not matched for sex. Bronchiectasis was present in at least two lobes per lung; none of the subjects had cystic bronchiectasis. Lung mass was significantly higher in the group with bronchiectasis compared to COPD $(\mathrm{p}=0.030)$ (table 2). In terms of emphysema, COPD lungs showed a higher percentage of lung with a density $<-950 \mathrm{HU}$ compared to control lungs $(\mathrm{p}<0.05)$, whereas no significant difference was found compared to COPD lungs with bronchiectasis (table 2).

More generations were counted in lungs of COPD with bronchiectasis and lungs of controls compared to COPD (both $\mathrm{p}<0.05$ ) (table 2, figure 2a). The number of bifurcations was higher in COPD lungs with bronchiectasis and control lungs compared to COPD lungs $(p<0.001)$ (figure $2 a)$. No difference in bifurcation numbers was present between lungs with bronchiectasis and control lungs. Interestingly, the airway diameter was not different between the groups $(p=0.23)$ (figure $2 b)$, unless the widest diameter per bifurcation was considered as a measure of lack of tapering $(p=0.010)$ (figure $2 c)$.

Numbers and diameters of airways on lobar microCT images

Characteristics of the patients on whose lungs lobar microCT was performed are summarised in table 3 . Figure 3 shows airway segmentation on lower lobes of a control lung, a COPD lung and a COPD lung with bronchiectasis. The higher resolution of these microCT scans, in comparison to the previously described CT evaluation, is reflected by the higher number of generations counted on these images (e.g. 22 and 19 versus 16 generations in lower lobes of control lung) and enables us to evaluate smaller airways in

\begin{tabular}{|c|c|c|c|c|}
\hline & Controls & COPD & COPD with bronchiectasis & p-value \\
\hline Subjects & 5 & 5 & 5 & \\
\hline Male/female & $4 / 1$ & $1 / 4$ & $5 / 0$ & 0.020 \\
\hline Age years & 60 (52-69) & $60(56-62)$ & $60(54-61)$ & 0.95 \\
\hline Height cm & 175 (164-178) & $161(157-169)$ & $173(173-179)^{*}$ & 0.031 \\
\hline $\mathrm{BMI} \mathbf{k g} \cdot \mathrm{m}^{-2}$ & $24.8(24.3-26.9)$ & $22.0(21.1-24.3)$ & $21.0(15.8-25.7)$ & 0.16 \\
\hline Smoking pack-years & $N A^{\#}$ & $30(30-38)$ & $39(28-65)$ & 0.55 \\
\hline Sputum production & NA & 1 & 4 & 0.058 \\
\hline Use of azithromycin & NA & 1 & 4 & 0.058 \\
\hline Exacerbations per year $n$ & NA & $2(1-3)$ & $3(2-4)$ & 0.41 \\
\hline \multicolumn{5}{|l|}{ FVC } \\
\hline L & NA & $1.83(1.66-2.07)$ & $2.48(2.02-3.47)$ & 0.032 \\
\hline$\%$ pred & NA & $65(52-77)$ & $61(48-79)$ & 1.00 \\
\hline \multicolumn{5}{|l|}{ FEV $_{1}$} \\
\hline L & NA & $0.83(0.60-0.86)$ & $0.90(0.62-1.18)$ & 0.73 \\
\hline$\%$ pred & NA & $30(28-34)$ & $22(15-33)$ & 0.31 \\
\hline FEV $1 / F V C$ & NA & $0.40(0.31-0.52)$ & $0.32(0.22-0.38)$ & 0.15 \\
\hline$D_{\text {Lco }} \%$ pred & NA & $34(32-45)$ & $28(21-43)$ & 0.42 \\
\hline Lobes with bronchiectasis $\mathrm{n}$ & NA & 0 & $2(2-2.5)$ & \\
\hline Modified Reiff score & NA & NA & $5(4-6)$ & \\
\hline
\end{tabular}

Data are presented as $\mathrm{n}$ or median (interquartile range), unless otherwise stated. Chi-squared, KruskalWallis with Dunn's post hoc test and Mann-Whitney tests were used. COPD: chronic obstructive pulmonary disease; BMI: body mass index; FVC: forced vital capacity; $\mathrm{FEV}_{1}$ : forced expiratory volume in $1 \mathrm{~s}$; $D_{\mathrm{Lco}}$ : diffusion capacity of the lung for carbon monoxide; NA: not applicable. ${ }^{\#}$ : these donors were registered as nonsmokers at the time of allocation. ${ }^{*}$ : $p<0.05$ compared to COPD. 
TABLE 2 Results of ex vivo computed tomography scans of the explanted lungs

\begin{tabular}{lcccc} 
& Control & COPD & COPD with bronchiectasis & p-value \\
\hline Left/right lung $\mathbf{n}$ & $0 / 5$ & $4 / 1$ & $3 / 2$ & \\
Total lung volume $\mathbf{L}$ & $3.5(2.7-3.6)$ & $3.1(2.6-3.4)$ & $4.2(3.5-5.0)$ & 0.10 \\
Lung mass g & $433(316-486)$ & $266(227-300)$ & $455(370-561)^{\#}$ & 0.030 \\
Lung density g. $\mathbf{L}^{-1}$ & $125(117-134)$ & $90(78-99)$ & $105(82-151)$ & 0.061 \\
Area <-950 HU \% & $17.9(11.2-22.1)$ & $44.0(35.4-49.4)^{*}$ & $31.2(14.4-47.5)$ & 0.027 \\
Airway generations $\mathbf{n}$ & $16(15-16.0)$ & $12(12-14)^{*}$ & $15(15-16)^{\#}$ & 0.011 \\
\hline
\end{tabular}

Data are presented as median (interquartile range), unless otherwise stated. p-values obtained by Kruskal-Wallis test. COPD: chronic obstructive pulmonary disease. Significant results found by Dunn's post hoc test: *: $p<0.05$ compared to control; * : p<0.05 compared to COPD.

more detail. The three-dimensional models of all lobar bronchial trees are shown in supplementary figure E1. The percentage area $<-950 \mathrm{HU}$ was similar in the upper lobes of COPD lungs with and without bronchiectasis, in contrast to the lower lobes, in which the COPD lungs with bronchiectasis seem to demonstrate a lower percentage of emphysema (table 3).

After segmentation, the COPD lungs with bronchiectasis showed considerably more airways in the upper and lower lobes compared to COPD lobes (table 3, figure 3, figure 4a,b). In comparison with control lobes, both COPD lobes without as well as with bronchiectasis had lower airway numbers in upper and lower lobes. This difference with controls was clearly less pronounced in COPD lobes with bronchiectasis (figure 4a,b). Throughout the first generations of the lower lobes, the diameters of COPD lungs with bronchiectasis were
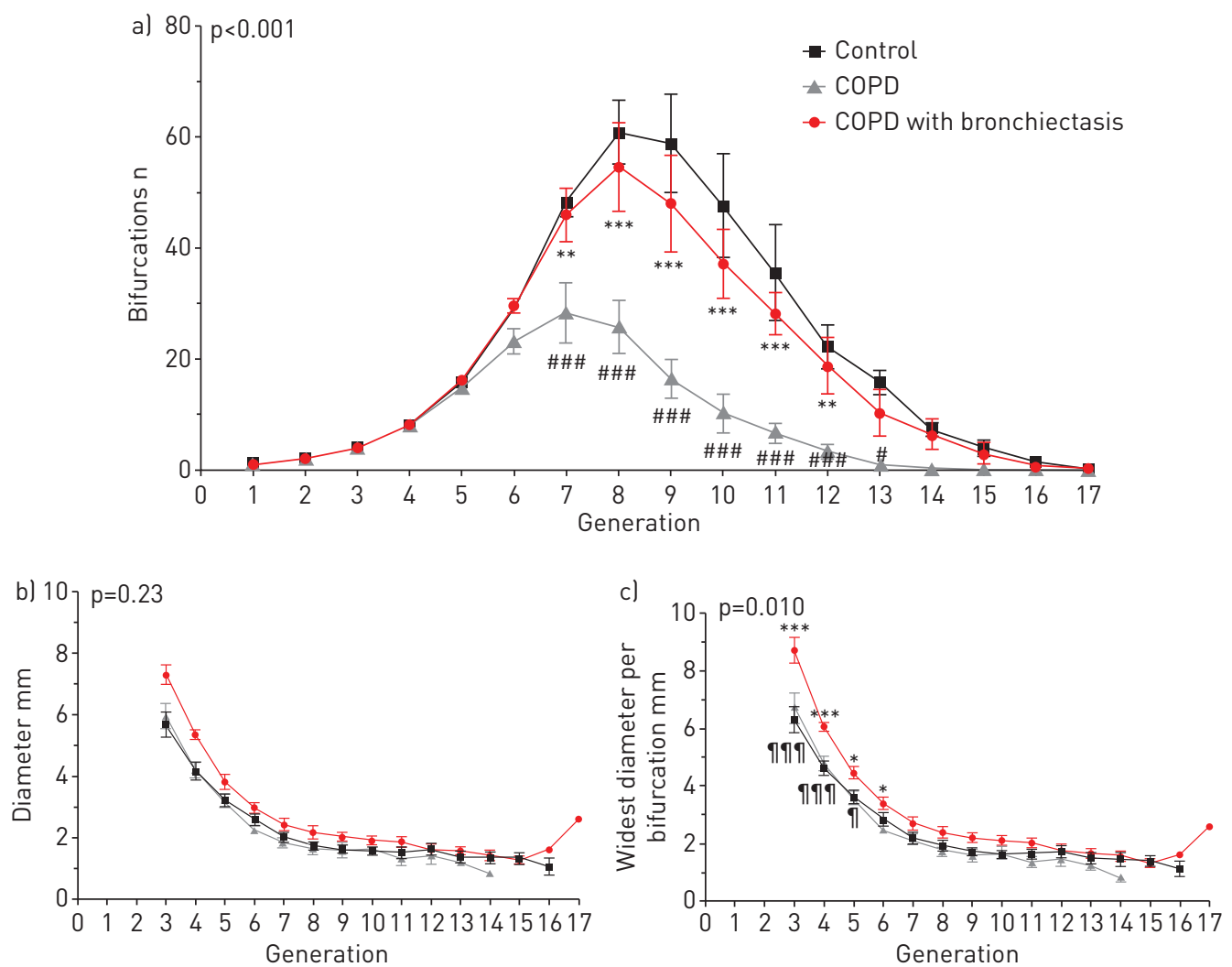

FIGURE 2 Bifurcation numbers and diameters on computed tomography (CT) images of frozen lungs. Results of measurements on CT images of control lungs $(n=5)$, chronic obstructive pulmonary disease (COPD) lungs $(n=5)$ and COPD lungs with bronchiectasis $(n=5)$. a) Number of bifurcations per generation; b) diameters of airway segments per generation; c) widest diameter per bifurcation, lack of tapering. Results are presented as mean \pm SEM. The reported $p$-value is the result of two-way ANOVA with symbols representing significant results of Tukey's post hoc test. *: $p<0.05,{ }^{* *}$ : $p<0.01,{ }^{* * *}$ : $p<0.001$ for COPD with bronchiectasis compared to COPD; \#: $p<0.05$, \#\#\#: $p<0.001$ for COPD compared to control; П: $p<0.05$, ๆाก: $p<0.001$ for COPD with bronchiectasis compared to control. 
TABLE 3 Characteristics of subjects and results of airway segmentation on lobar micro-computed tomography

\begin{tabular}{|c|c|c|c|c|c|c|}
\hline \multirow[b]{2}{*}{ Sex } & \multicolumn{2}{|c|}{ Control } & \multicolumn{2}{|c|}{ COPD } & \multicolumn{2}{|c|}{$\begin{array}{c}\text { COPD with } \\
\text { bronchiectasis }\end{array}$} \\
\hline & M & M & M & M & M & M \\
\hline Age years & 58 & 57 & 64 & 64 & 61 & 65 \\
\hline Height cm & 185 & 180 & 170 & 168 & 173 & 170 \\
\hline Weight kg & 95 & 80 & 70 & 62 & 78 & 60 \\
\hline $\mathrm{BMI} \mathrm{kg} \cdot \mathrm{m}^{-2}$ & 27.8 & 24.7 & 24.2 & 22.0 & 26.0 & 20.8 \\
\hline Smoking pack-years & NA" & $N A^{\pi}$ & 40 & 40 & 50 & 40 \\
\hline \multicolumn{7}{|l|}{ FVC } \\
\hline L & NA & NA & 3.18 & 1.65 & 2.48 & 3.70 \\
\hline$\%$ pred & NA & NA & 84 & 45 & 61 & 97 \\
\hline \multicolumn{7}{|l|}{ FEV $_{1}$} \\
\hline L & NA & NA & 0.82 & 0.88 & 0.48 & 1.38 \\
\hline$\%$ pred & NA & NA & 28 & 30 & 15 & 46 \\
\hline $\mathrm{FEV}_{1} / \mathrm{FVC}$ & NA & NA & 0.26 & 0.53 & 0.19 & 0.37 \\
\hline$D_{\text {Lco }} \%$ pred & NA & NA & 32 & 34 & 38 & 28 \\
\hline Lobes with bronchiectasis $\mathrm{n}$ & NA & NA & 0 & 0 & 2 & 3 \\
\hline Modified Reiff score & NA & NA & NA & NA & 4 & 4 \\
\hline Right/left lung & Right & Left & Right & Left & Right & Left \\
\hline \multicolumn{7}{|l|}{ Upper lobe ${ }^{\#}$} \\
\hline Volume L & NA & NA & 2.477 & 1.697 & 2.095 & 1.863 \\
\hline Area $<-950 \mathrm{HU} \%$ & NA & NA & 38 & 40 & 37 & 35 \\
\hline Generations n & 19 & 19 & 14 & 14 & 14 & 18 \\
\hline Airways n & 1478 & 958 & 324 & 224 & 602 & 628 \\
\hline \multicolumn{7}{|l|}{ Lower lobe } \\
\hline Volume L & NA & NA & 1.625 & 1.317 & 1.329 & 0.847 \\
\hline Area $<-950 \mathrm{HU} \%$ & NA & NA & 33 & 38 & 24 & 23 \\
\hline Generations n & 22 & 19 & 19 & 17 & 20 & 17 \\
\hline Airways n & 1350 & 1129 & 364 & 384 & 683 & 926 \\
\hline
\end{tabular}

Individual results are presented. COPD: chronic obstructive pulmonary disease; M: male; BMI: body mass index; FVC: forced vital capacity; $F E V_{1}$ : forced expiratory volume in $1 \mathrm{~s} ; D_{\mathrm{Lco}}$ : diffusing capacity of the lung for carbon monoxide; NA: not applicable. ${ }^{\#}$ : sum of upper and middle lobe in case of right lung; " : these donors were registered as nonsmokers at the time of allocation.

wider compared to COPD lungs and narrower in COPD lungs compared to control ( $\mathrm{p}=0.010)$ (figure 3, figure $4 \mathrm{~d})$. This difference was not present in the upper lobes (figure $4 \mathrm{c})(\mathrm{p}=0.30)$. If the airways in the lower lobes were subdivided by diameter of more or less than $2 \mathrm{~mm}$, the difference between COPD lungs with and without bronchiectasis was maintained, but particularly present in larger airways $(>2 \mathrm{~mm})$, whereas the difference between COPD and control was more pronounced in the small airways $(\leqslant 2 \mathrm{~mm})(\mathrm{p}<0.001)$ (figure $4 \mathrm{e}, \mathrm{f}$ ).

Tissue characteristics and terminal bronchioles on microCT images of frozen tissue cores Tissue percentage, surface density and number of terminal bronchioles per millilitre was significantly lower in both COPD groups compared to controls (supplementary figure E2a-c). Between tissue cores of COPD patients with or without bronchiectasis, no difference was found for these variables. Moreover, the number and diameter of terminal bronchioles per millilitre was similar between these groups (supplementary figure E2a-d and supplementary table E1). Taking spatial distribution into account, no difference was found in any of the groups between cores from upper versus lower lobes, nor between cores from regions with bronchiectasis and regions without bronchiectasis (supplementary table E2).

\section{Discussion}

To our knowledge, this is the first study investigating and comparing the entire bronchial tree in end-stage COPD lungs with and without bronchiectasis and the first report on microCT of large lung specimens. On the lower resolution clinical CT, we found that COPD with bronchiectasis showed little reduction in airway numbers, which contrasted with COPD without bronchiectasis, which showed extensive reduction in airway numbers, both compared to controls. When measured on the higher resolution microCT images of whole lobes, COPD with bronchiectasis showed only a moderate reduction in the large and small airways compared to controls. At the same time, the reduction in the terminal bronchioles was equal to what was found in COPD without bronchiectasis. 

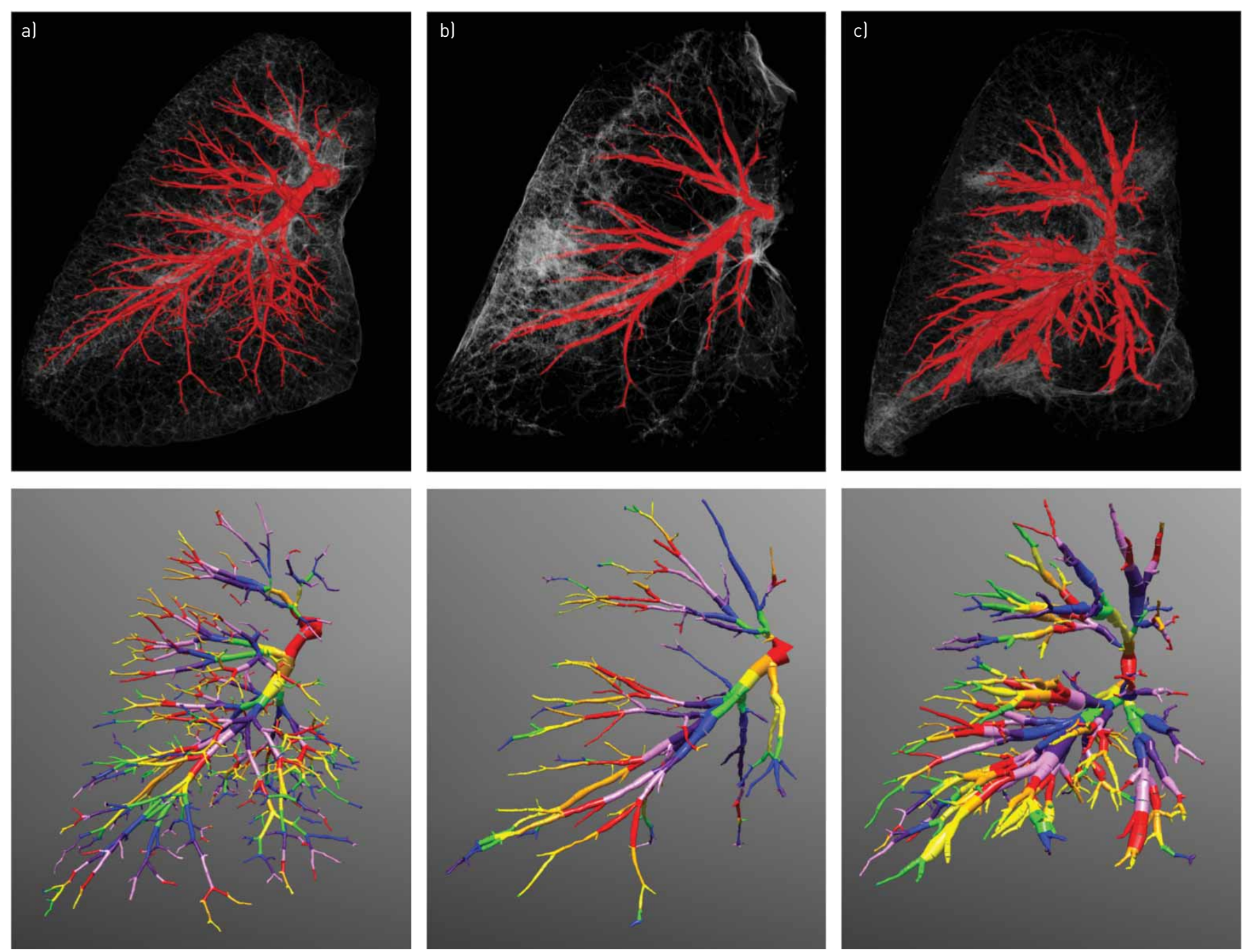

FIGURE 3 Airway segmentation of lower lobes on micro-computed tomography images. Lower lobes of a) control lung, b) chronic obstructive pulmonary disease (COPD) lung and c) COPD lung with bronchiectasis. The upper panels show the bronchial tree after segmentation, projected on the contours of the lobe. The lower panels show the same bronchial tree after automated annotation, with each colour representing a different generation within the lobe.

The finding of more airways in COPD lungs with bronchiectasis compared to COPD lungs without bronchiectasis suggests less severe emphysema at the moment of transplantation [15, 27]. However, all patients had end-stage COPD, meeting the criteria for lung transplantation [20] without significant difference in forced expiratory volume in $1 \mathrm{~s}\left(\mathrm{FEV}_{1}\right), \mathrm{FEV}_{1} /$ forced vital capacity $(\mathrm{FVC})$ ratio or diffusion capacity of the lung for carbon monoxide. In addition, the degree of emphysema measured using percentage of area $<-950 \mathrm{HU}$, a threshold commonly used to quantify emphysema on CT, or the surface density measured on microCT of tissue cores was not different between the groups. All together, these parameters indicate that our observation of higher airway counts in lungs with bronchiectasis compared to COPD alone, could not be attributed to different disease severity.

One explanation for the higher number of airways in bronchiectasis could be a higher visibility because of increased airway diameter, as previously postulated in cystic fibrosis [28]. However, our data do not fully support this hypothesis, as the number of airways in the lower lobes was significantly higher in bronchiectasis from generation 10 onwards, whereas diameters were mainly wider in the preceding generations. Furthermore, the increased airway count was not related to the presence of bronchiectasis, since upper lobes (which were less or not affected by bronchiectasis) also showed this difference.

An alternative explanation for the higher airway counts could be found in less pronounced airway loss in COPD lungs that develop bronchiectasis over time. However, it has been shown that airway loss occurs from the early stages of the disease $[15-17,29,30]$, whereas bronchiectasis seems to be associated with more advanced stages of COPD $[12,13]$. Unfortunately, the use of end-stage disease lungs prevents us from drawing conclusions about early disease stages. 

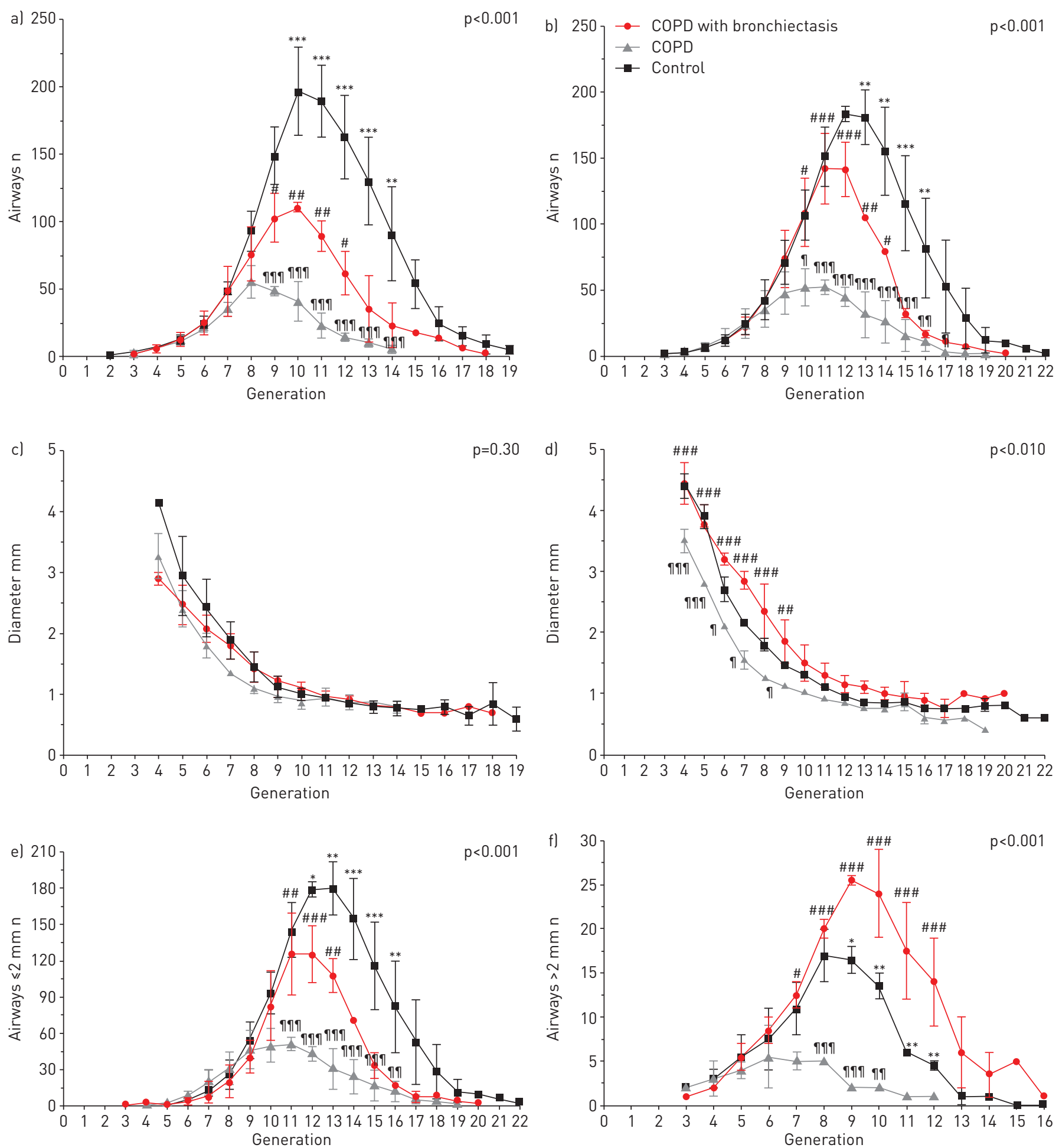

FIGURE 4 Airway numbers and diameters as calculated on segmented airway models obtained by micro-computed tomography (microCT) of air-dried lobes. Results of measurements on microCT of control lobes ( $n=2$ upper and $n=2$ lower lobes), chronic obstructive pulmonary disease (COPD) lobes ( $n=2$ upper and $n=2$ lower lobes) and COPD lobes with bronchiectasis ( $n=2$ upper and $n=2$ lower lobes). a, b) numbers of airways per generation; $c, d$ ) average diameters of airway segments per generation in upper ( $a, c)$ and lower (b, d) lobes. e, f) Airways in the lower lobes were separated based on diameter $\leqslant 2 \mathrm{~mm}$ and $>2 \mathrm{~mm}$, respectively. Data are presented as mean \pm SEM. The reported $p$-value is the result of two-way ANOVA with symbols representing significant results of Tukey's post hoc test. *: $p<0.05,{ }^{* *}: p<0.01$ and ${ }^{* * *}$ : $p<0.001$, for COPD with bronchiectasis

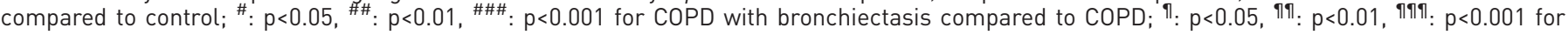
COPD compared to control. 
Even more intriguing would be to hypothesise that COPD patients with bronchiectasis have a more differentiated bronchial tree from early life onwards, which could predispose them to development of bronchiectasis rather than to loss of airways. Recently, the importance of early life events on lung structure and function is well accepted [31,32]. Variation in segmental airway branching [33], lung structure on CT [29] (both genetically determined) and reduced total airway count [17] have been suggested as risk factors for COPD. A lower total airway count is associated with airflow limitation, independently of emphysema and smoking, which suggests that the composition of the airway tree may determine maximum attained lung function [17]. Our data add to the idea that the composition of the bronchial tree is also relevant in the development of specific phenotypes of respiratory disease. The sequence in which emphysema and bronchiectasis develop in patients with COPD is still unclear and there is obviously need for longitudinal imaging studies to answer these questions. Investigating lungs diagnosed with bronchiectasis before the development of COPD due to smoking, would also be valuable.

An important strength of the study is the use of microCT on whole lung lobes. We found reduced airway counts in the zone between generation 10 and the (pre-)terminal bronchioles, whereas previous studies only focused on the extremities of the bronchial tree. Furthermore, this study strengthens the idea that imaging may serve as a useful biomarker in COPD, as recently highlighted in this journal [34].

This study suffers from a number of important limitations. First, the relevance of bronchiectasis in COPD has been questioned because diagnostic criteria may overestimate bronchodilation $[35,36]$ and higher bronchoarterial ratios have been attributed to reduced vessel size rather than increased bronchial diameters in smokers [37]. Unfortunately, our approach did not allow for quantification of vessel dimensions. Second, the use of rejected lungs as healthy controls may be questioned. Fortunately, most lungs were not declined because of poor quality but because of other, nonparenchymal reasons. Even if we assume that the intensive care period (prior to organ donation) would affect lung quality, it would not affect the investigated variables such as airway counts. Third, lungs in the conventional CT analysis were not matched for sex, side (left/right) or FVC. We tried to confirm our results in the lobar microCT analysis in which we could match for sex and location, but not for lung function variables. This illustrates the inherent heterogeneity of COPD, even in end-stage disease. Fourth, pre-lung transplant follow-up was not standardised in patients referred from different hospitals, which precludes us from a thorough comparison of clinical characteristics. Nevertheless, patients with bronchiectasis had a distinct clinical syndrome based on the use of azithromycin and the presence of sputum. Moreover, the lungs with bronchiectasis had a modified Reiff score of $\geqslant 4$, meaning that four or more lobes had tubular bronchiectasis. Finally, sample sizes are low, given the limited availability of unused donor lungs and of COPD lungs with bronchiectasis. The pronounced visual differences in a limited sample size are still striking and should therefore lead to future longitudinal studies focusing on associations between COPD, bronchiectasis, emphysema and initial airway counts.

In conclusion, our study shows a bronchial tree with more airway branches in end-stage COPD lungs with bronchiectasis compared to COPD lungs without bronchiectasis, despite similar degree of emphysema and loss of terminal bronchioles. Our findings indicate that the intrinsic composition of the airway tree may predispose to certain phenotypes in COPD.

Acknowledgements: We would like to thank the entire lung transplant team of UZ Leuven, Belgium; Robin Vos, the transplant coordinators and the surgeons of the thoracic surgery unit (Dirk Van Raemdonck, Willy Coosemans, Paul De Leyn, Philippe Nafteux, Hans Van Veer, Lieven Depypere and Herbert Decaluwé) for their collaboration in collecting the explanted lungs; and the pneumology research unit for their help in collecting and processing lungs.

Support statement: This study was supported by the KU Leuven Research Fund (C24/15/030) and the AstraZeneca Chair in respiratory pathophysiology KU Leuven. The Ghent University Special Research Fund (BOF-UGent) is acknowledged for the financial support to the Centre of Expertise UGCT (BOF.EXP.2017.000007). Funding information for this article has been deposited with the Crossref Funder Registry.

Conflict of interest: S. Everaerts reports grants from FWO, Flemish research fund (11V9417N), during the conduct of the study. J.E. McDonough has nothing to disclose. S.E. Verleden has nothing to disclose. I. Josipovic has nothing to disclose. M. Boone has nothing to disclose. A. Dubbeldam has nothing to disclose. C. Mathyssen has nothing to disclose. J. Serré has nothing to disclose. L.J. Dupont has nothing to disclose. G. Gayan-Ramirez has nothing to disclose. J. Verschakelen has nothing to disclose. J.C. Hogg has nothing to disclose. G.M. Verleden has nothing to disclose. B.M. Vanaudenaerde has nothing to disclose. W. Janssens reports grants from AstraZeneca, Chiesi and Boehringer Ingelheim, outside the submitted work.

\section{References}

1 Rabe KF, Watz H. Chronic obstructive pulmonary disease. Lancet 2017; 389: 1931-1940.

2 Han MK, Agusti A, Calverley PM, et al. Chronic obstructive pulmonary disease phenotypes: the future of COPD. Am J Respir Crit Care Med 2010; 182: 598-604. 


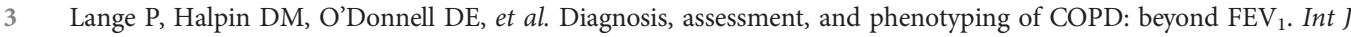
Chron Obstruct Pulmon Dis 2016; 11: Special issue, 3-12.

4 Pasteur MC, Bilton D, Hill AT. British Thoracic Society guideline for non-CF bronchiectasis. Thorax 2010; 65: Suppl. 1, i1-i58.

5 Naidich DP, McCauley DI, Khouri NF, et al. Computed tomography of bronchiectasis. J Comput Assist Tomogr 1982; 6: 437-444.

6 Martinez-Garcia MA, Miravitlles M. Bronchiectasis in COPD patients: more than a comorbidity? Int J Chron Obstruct Pulmon Dis 2017; 12: 1401-1411.

7 Lonni S, Chalmers JD, Goeminne PC, et al. Etiology of non-cystic fibrosis bronchiectasis in adults and its correlation to disease severity. Ann Am Thorac Soc 2015; 12: 1764-1770.

8 Quint JK, Millett ERC, Joshi M, et al. Changes in the incidence, prevalence and mortality of bronchiectasis in the UK from 2004 to 2013: a population-based cohort study. Eur Respir J 2016; 47: 186-193.

9 McDonnell MJ, Aliberti S, Goeminne PC, et al. Comorbidities and the risk of mortality in patients with bronchiectasis: an international multicentre cohort study. Lancet Respir Med 2016; 4: 969-979.

10 Martínez-García M-A, de la Rosa Carrillo D, Soler-Cataluña J-J, et al. Prognostic value of bronchiectasis in patients with moderate-to-severe chronic obstructive pulmonary disease. Am J Respir Crit Care Med 2013; 187: 823-831.

11 Jairam PM, van der Graaf Y, Lammers J-WJ, et al. Incidental findings on chest CT imaging are associated with increased COPD exacerbations and mortality. Thorax 2015; 70: 725-731.

12 Ni Y, Shi G, Yu Y, et al. Clinical characteristics of patients with chronic obstructive pulmonary disease with comorbid bronchiectasis: a systemic review and meta-analysis. Int J Chron Obstruct Pulmon Dis 2015; 10: 1465-1475.

13 Du Q, Jin J, Liu X, et al. Bronchiectasis as a comorbidity of chronic obstructive pulmonary disease: a systematic review and meta-analysis. PLoS One 2016; 11: e0150532.

14 Hogg JC, Chu F, Utokaparch S, et al. The nature of small-airway obstruction in chronic obstructive pulmonary disease. N Engl J Med 2004; 350: 2645-2653.

15 McDonough JE, Yuan R, Suzuki M, et al. Small-airway obstruction and emphysema in chronic obstructive pulmonary disease. N Engl J Med 2011; 365: 1567-1575.

16 Ding M, Chen Y, Guan W-J, et al. Measuring airway remodeling in patients with different COPD staging using endobronchial optical coherence tomography. Chest 2016; 150: 1281-1290.

17 Kirby M, Tanabe N, Tan WC, et al. Total airway count on computed tomography and the risk of chronic obstructive pulmonary disease progression. Findings from a population-based study. Am J Respir Crit Care Med 2018; 197: 56-65.

18 Whitwell F. A study of the pathology and pathogenesis of bronchiectasis. Thorax 1952; 7: 213-239.

19 Roberts HR, Wells AU, Milne DG, et al. Airflow obstruction in bronchiectasis: correlation between computed tomography features and pulmonary function tests. Thorax 2000; 55: 198-204.

20 Weill D, Benden C, Corris PA, et al. A consensus document for the selection of lung transplant candidates: 2014 - an update from the Pulmonary Transplantation Council of the International Society for Heart and Lung Transplantation. J Heart Lung Transplant 2015; 34: 1-15.

21 Chalmers JD, Goeminne P, Aliberti S, et al. The bronchiectasis severity index. An international derivation and validation study. Am J Respir Crit Care Med 2014; 189: 576-585.

22 Verleden SE, Vasilescu DM, Willems S, et al. The site and nature of airway obstruction after lung transplantation. Am J Respir Crit Care Med 2014; 189: 292-300.

23 Vasilescu DM, Phillion AB, Tanabe $\mathrm{N}$, et al. Nondestructive cryomicro-CT imaging enables structural and molecular analysis of human lung tissue. J Appl Physiol 2017; 122: 161-169.

24 Masschaele B, Dierick M, Van Lo D, et al. HECTOR: a $240 \mathrm{kV}$ micro-CT setup optimized for research. J Phys Conf Ser 2013; 463: 012012.

25 Yushkevich PA, Piven J, Hazlett HC, et al. User-guided 3D active contour segmentation of anatomical structures: significantly improved efficiency and reliability. NeuroImage 2006; 31: 1116-1128.

26 Wearne SL, Rodriguez A, Ehlenberger DB, et al. New techniques for imaging, digitization and analysis of three-dimensional neural morphology on multiple scales. Neuroscience 2005; 136: 661-680.

27 Diaz AA, Valim C, Yamashiro T, et al. Airway count and emphysema assessed by chest CT imaging predicts clinical outcome in smokers. Chest 2010; 138: 880-887.

28 Boon M, Verleden SE, Bosch B, et al. Morphometric analysis of explant lungs in cystic fibrosis. Am J Respir Crit Care Med 2016; 193: 516-526.

29 Oelsner EC, Ortega VE, Smith BM, et al. A genetic risk score associated with COPD susceptibility and lung structure on computed tomography. Am J Respir Crit Care Med 2019; 200: 721-731.

30 Koo H-K, Vasilescu DM, Booth S, et al. Small airways disease in mild and moderate chronic obstructive pulmonary disease: a cross-sectional study. Lancet Respir Med 2018; 6: 591-602.

31 Lange P, Celli B, Agustí A, et al. Lung-function trajectories leading to chronic obstructive pulmonary disease. N Engl J Med 2015; 373: 111-122.

32 Martinez FD. Early-life origins of chronic obstructive pulmonary disease. N Engl J Med 2016; 375: 871-878.

33 Smith BM, Traboulsi H, Austin JHM, et al. Human airway branch variation and chronic obstructive pulmonary disease. Proc Natl Acad Sci USA 2018; 115: E974-E981.

34 Washko GR, Parraga G. COPD biomarkers and phenotypes: opportunities for better outcomes with precision imaging. Eur Respir J 2018; 52: 1801570.

35 Lynch DA, Newell JD, Tschomper BA, et al. Uncomplicated asthma in adults: comparison of CT appearance of the lungs in asthmatic and healthy subjects. Radiology 1993; 188: 829-833.

36 Diaz AA, Young TP, Maselli DJ, et al. Bronchoarterial ratio in never-smokers adults: implications for bronchial dilation definition. Respirology 2017; 22: 108-113.

37 Diaz AA, Young TP, Maselli DJ, et al. Quantitative CT measures of bronchiectasis in smokers. Chest 2017; 151: $1255-1262$ 